\title{
Benzotriazole as an inhibitor of the anodic dissolution of cadmium, bismuth, and their eutectic alloy in a neutral borate solution
}

\author{
A. G. Berezhnaya,* V. I. Mishurov and V. V. Ekilik \\ Southern Federal University, ul. Zorge 7, Rostov-on-Don, 344090 Russian Federation \\ *E-mail:ber@sfedu.ru
}

\begin{abstract}
The electrochemical behavior of an eutectic cadmium-bismuth alloy in borate buffer solution containing 1,2,3-benzotriazole (BTA) as an additive has been studied. The additive inhibits the cadmium and alloy dissolution to an appreciable extent. It has been shown that the experimental polarization curve of the alloy is similar to that calculated based on the additive scheme. However, the currents on the experimental curve are lower due to selective mode of cadmium dissolution from the alloy. The kinetics of passive film formation was also studied.
\end{abstract}

Key words: bismuth, cadmium, eutectic alloy, anodic dissolution, inhibitor, benzotriazole.

Received: March 7, 2013.

doi: 10.17675/2305-6894-2013-2-2-132-137

\section{Introduction}

The effect of surface-active substances on the regularities of electrochemical dissolution of heterogeneous alloys has been studied less thoroughly than for homogeneous alloys. $[1,2]$. Benzotriazole is known as an efficient complexing agent for non-ferrous metals [3,4]. The surface or bulk complexes that are formed can change the characteristics of the polarization curves of cadmium, bismuth and their eutectic alloy.

\section{Experimental}

Electrochemical measurements were carried out by common techniques in a threeelectrode cell. Forward polarization curves in the direction of potential $(E)$ increase from $-1 \mathrm{~V}$ to $1.5 \mathrm{~V}$ and reverse curves in the same potential range were recorded using stepwise $E$ variation with a step of $50 \mathrm{mV}$ and exposure for 1 min at each $E$ value. In addition, anodic chronoamperograms in the passive region at $E=0.5$ and $0.9 \mathrm{~V}$ as well as cathodic chronopotentiograms were recorded. A saturated silver chloride electrode was used as the reference and the $E$ values are reported with respect to it. Electrodes with a disc-shaped working surface (area $=0.4 \mathrm{~cm}^{2}$ ) were mechanically abraded and polished. The studies were carried out in borate buffer solutions with and without $0.1 \mathrm{mM}$ of 1,2,3-benzotriazole. The efficiency was estimated by inhibition factor $\gamma$, which is the ratio of the process rates in the pure solution and with the additive. 


\section{Results and Discussion}

Benzotriazole affects the electrochemical behavior of cadmium and bismuth in a different way. If polarization is performed from electropositive potentials to anodic ones, BTA inhibits the cathodic evolution of hydrogen on bismuth, stimulates it on cadmium, and changes the corrosion potentials $E_{\text {cor }}$, Table 1 . The process is inhibited more strongly on the eutectic alloy having a fine-dispersed structure. In borate solution with BTA, the $E_{\text {cor }}$ value on the alloy is close to that of cadmium and significantly below than that of bismuth.

Table 1. Effect of benzotriazole on the characteristics of anodic polarization curves

\begin{tabular}{|c|c|c|c|c|c|c|c|c|c|c|}
\hline \multirow{2}{*}{$\begin{array}{l}{[\mathbf{B i}]_{0}} \\
\text { wt. } \%\end{array}$} & \multirow{2}{*}{$-\Delta E_{\text {cor }}, \mathrm{V}$} & \multirow{2}{*}{$-\Delta E_{\mathrm{ap}, 1}, \mathrm{~V}$} & \multirow{2}{*}{$\Delta E_{\mathrm{ap}, 2}, \mathrm{~V}$} & \multirow{2}{*}{$\gamma_{\mathrm{ap}, 1}$} & \multirow{2}{*}{$\gamma_{\mathrm{ap}, 2}$} & \multicolumn{5}{|c|}{$\gamma$ at $E, V$} \\
\hline & & & & & & -0.9 & -0.3 & 0.5 & 1.0 & 1.5 \\
\hline 0 & 0.15 & 0.05 & 0 & 6.2 & 1.1 & 0.5 & 1.3 & 4.1 & 6.0 & 11 \\
\hline 60 & 0.05 & 0 & - & 37.7 & - & 4.4 & 3.6 & 6.8 & 7.0 & 15.7 \\
\hline 100 & -0.05 & - & - & - & - & 2.2 & 4.4 & 1.2 & 1.0 & - \\
\hline
\end{tabular}

Note: $\Delta E_{\text {cor }}=E_{\text {cor }}^{0}-E_{\text {cor }}^{\text {add }}$, where $E_{\text {cor }}^{0}$ and $E_{\text {cor }}^{\text {add }}$ are the corrosion potentials in solutions with and without BTA, respectively. A dash means that the parameter if not available for the corresponding curve.

Benzotriazole practically does not change the form of the anodic curve on bismuth. The process rate in the presence of the additive decreases on all the characteristic parts of the curve, Fig. 1, Table 1. In comparison with pure borate solution, BTA changes the shape of the anodic polarization curves on cadmium and on the alloy. The potentials of the first $E_{\mathrm{ap}, 1}$ and second $E_{\mathrm{ap}, 2}$ anodic peaks in the solution without and with the additive are similar. The process rate at $E_{\text {ap }, 1}$ decreases essentially but practically does not change at $E_{\text {ap }, 2}$. This results in a bend on the curve on cadmium.

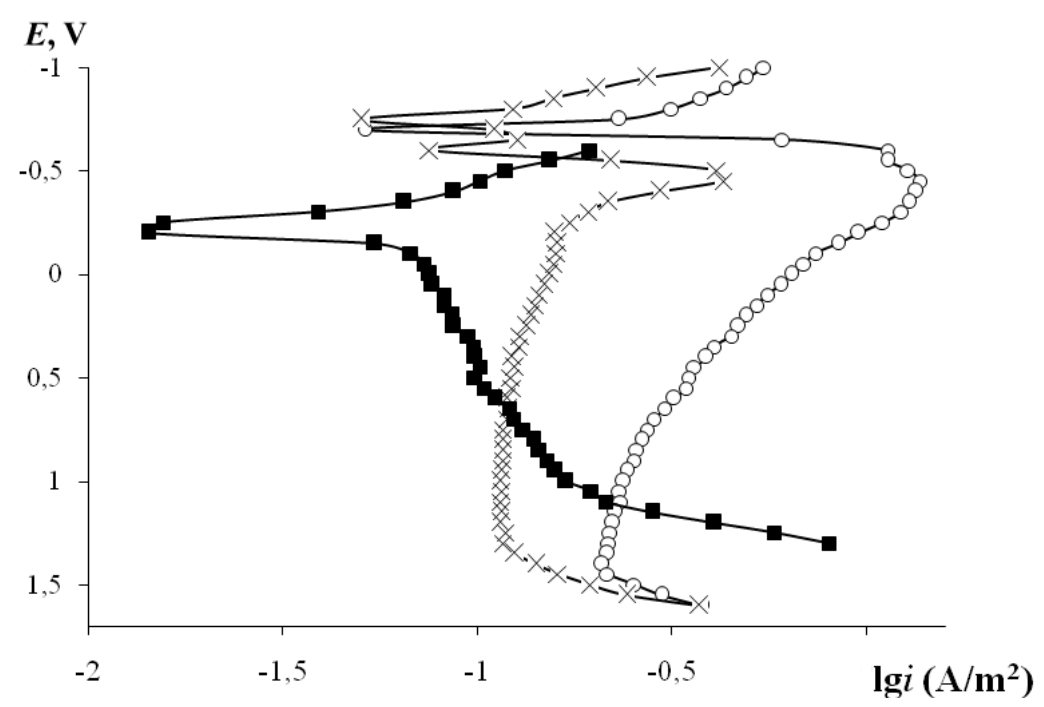

Fig. 1. Polarization curves on cadmium $(\circ)$, bithmus $(\mathbf{\square})$, and their eutectic alloy $(\times)$ in borate solution in the presence of 1,2,3-benzotriazole. 
A clear two-peak anodic curve is observed on the alloy. The process rate at $E_{\mathrm{ap}, 1}$ is four times smaller than that at $E_{\mathrm{ap}, 2}$. Transition to a stable passive state on cadmium and eutectic alloy is difficult and continues up to the oxygen evolution potentials.

Spectrophotometric analysis of solutions containing $\mathrm{Cd}^{2+}$ ions and benzotriazole ligands showed no complexation to occur. Obviously, BTA is adsorbed on the cadmium surface, preventing oxygen chemisorption and formation of an oxide-hydroxide passive film. The inhibition factor $\gamma$ increases with an increase in potential.

The experimental curve on the alloy was compared with the theoretical curve generated by the additive scheme:

$$
i_{\text {theor }}=i_{\mathrm{Cd}} N_{\mathrm{Cd}}+i_{\mathrm{Bi}} N_{\mathrm{Bi}}
$$

The $E_{\text {cor }}$ on the experimental curve is close to the calculated value. The dissolution rates of the alloy in a solution without the additive on the experimental and theoretical curves match. In a solution containing BTA, the theoretical dissolution rate in two or more times higher. Probably, the finely dispersed phase of cadmium in the alloy is more reactive and better adsorbs the additive. Furthermore, BTA may be retained in the pores formed.

Cadmium, bismuth and their alloy do not undergo local damage in the presence of BTA. The process rates on the reverse curves are much lower than on the direct ones. The additive changes the characteristics of the reverse polarization curves. The $E_{\text {cor }}$ and cathodic currents are higher than on the forward curves, whereas the anodic currents are smaller. Upon reverting the polarization direction, BTA decreases the rates of cathodic and anodic reactions on $\mathrm{Cd}$ and on the alloy but increases them on bismuth, Table 2 .

Table 2. Dependence of the inhibition factor (reverse polarization) on potential.

\begin{tabular}{cccccc}
\hline $\begin{array}{c}{[\mathbf{B i}]_{\mathbf{0}},} \\
\mathbf{w t . \%}\end{array}$ & $\mathbf{- 0 . 8}$ & $\mathbf{- 0 . 1}$ & $\mathbf{0 . 5}$ & $\mathbf{1 . 0}$ & $\mathbf{1 . 5}$ \\
\cline { 2 - 6 } & 10.9 & 20.1 & 15.8 & 11.6 & 9.92 \\
\hline 0 & 2.86 & 32.5 & 15.8 & 10.0 & 13.4 \\
60 & 0.40 & 0.67 & 0.67 & 0.45 & - \\
\hline
\end{tabular}

The $\gamma$ values substantially exceed the inhibition factors obtained on the direct curves. This may be related to an improvement in the properties of the passive film.

The current was observed to decrease with time on the chronoamperograms on cadmium, bismuth, and alloy. In contrast to the pure borate soluton, dissolution is steady it time [5]. Linear sections are also observed on the curves in $i^{-1}, \tau^{1 / 2}$ coordinates. These sections correspond to the equations:

$$
\begin{gathered}
i^{-1}=\alpha_{1}^{-1}\left(\tau^{1 / 2}-\tau_{0}^{1 / 2}\right) \\
i^{-1}=i_{0}^{-1}+\alpha_{2}^{-1} \tau^{1 / 2}
\end{gathered}
$$


The applicability ranges of equations (1) and (2) and their parameters are presented in Table 3.

Table 3. Correlation coefficients $R$, range of applicability $\tau$, and parameters of equations (1) and (2) as a function of $E$.

\begin{tabular}{|c|c|c|c|c|c|c|c|c|}
\hline \multirow{2}{*}{$\begin{array}{l}{[\mathrm{Bi}]_{0},} \\
\text { wt.\% }\end{array}$} & \multicolumn{4}{|c|}{ Parameters of Equations (1) } & \multicolumn{4}{|c|}{ Parameters of Equations (2) } \\
\hline & $\tau_{0}, \mathbf{s}$ & $\tau, \mathbf{s}$ & $\alpha_{1}^{-1}$ & $R, \%$ & $\tau, \mathbf{s}$ & $i_{0}^{-1}$ & $\alpha_{2}^{-1}$ & $R, \%$ \\
\hline \multicolumn{9}{|c|}{$E=0.5 \mathrm{~V}$} \\
\hline \multirow{2}{*}{0} & \multirow{2}{*}{65} & \multirow{2}{*}{$900-1500$} & \multirow{2}{*}{0.05} & \multirow{2}{*}{99.0} & $0-40$ & 0.15 & 0.11 & 96.0 \\
\hline & & & & & 40-900 & 0.68 & 0.02 & 99.8 \\
\hline \multirow{3}{*}{60} & 1 & $0-60$ & 0.13 & 99.9 & \multirow{3}{*}{$60-1080$} & \multirow{3}{*}{0.57} & \multirow{3}{*}{0.05} & \multirow{3}{*}{99.6} \\
\hline & 190 & $1080-1500$ & 0.11 & 99.6 & & & & \\
\hline & 285 & $600-1500$ & 0.62 & 99.7 & & & & \\
\hline 100 & - & - & - & - & $0-1500$ & 0.28 & 0.56 & 99.8 \\
\hline \multicolumn{9}{|c|}{$E=0.9 \mathrm{~V}$} \\
\hline \multirow{2}{*}{0} & \multirow{2}{*}{14} & \multirow{2}{*}{$780-1500$} & \multirow{2}{*}{0.10} & \multirow{2}{*}{98.8} & $0-40$ & 0.05 & 0.14 & 98.7 \\
\hline & & & & & $40-780$ & 0.68 & 0.03 & 99.3 \\
\hline \multirow{2}{*}{60} & 1 & $0-60$ & 0.10 & 99.3 & \multirow{2}{*}{$60-1320$} & \multirow{2}{*}{0.87} & \multirow{2}{*}{0.03} & \multirow{2}{*}{99.6} \\
\hline & 15 & $1320-1500$ & 0.12 & 99.9 & & & & \\
\hline 100 & 1 & $0-1500$ & 0.49 & 99.8 & - & - & - & - \\
\hline
\end{tabular}

Note: $i_{0}^{-1}$ and $\alpha^{-1}$ values are expressed in $\mathrm{m}^{2} / \mathrm{A}$ and $\mathrm{m}^{2} /\left(\mathrm{A} \cdot \mathrm{sec}^{1 / 2}\right)$, respectively.

In this case, differences exist due to competitive adsorption of the additive on the surface and its different effect on the dissolution of the components in the case of the alloy. In a solution without BTA, the chronoamperograms on cadmium were described only by Equation (2) with different constants within different time intervals, those of $\mathrm{Bi}$ were described only by Equation (1), and those of the eutectic at $E=0.5 \mathrm{~V}$ by Equation (1) and at $E=0.9 \mathrm{~V}$, both by Equations (1) and (2). In the presence of BTA, long sections corresponding to both equations were observed on the chronoamperograms. The larger $\alpha^{-1}$ values on bismuth are probably due to the smaller diffusion coefficient in the film.

The process rate decreases with time both in the pure solution and in the presence of BTA, which may indicate the absence of apparent phase formation on the surface [6]. In the initial period of time, the additive behaves as an inhibitor. As the exposure time increases, the inhibition effect increases on cadmium and decreases on bismuth. The $\gamma$ values become closer to those obtained in polarization measurements. It may indirectly 
indicate that the composition of the surface layer is similar to that of the passive films formed during the time when the polarization curves were recorded.

In the course of time, inhibition of the alloy is replaced by stimulation. The $\gamma$ values are lower than those obtained in the polarization measurements. The cause of this change in $\gamma$ with time lies in the finely dispersed structure of the eutectic providing a higher dissolution rate than on cadmium at the initial stages. The high degree of surface renewal with simultaneous dissolution of the alloy components, as well as competing adsorption of BTA and oxygen, prevent the formation of a passive layer.

Cathodic chronopotentiograms of the metals and the alloy obtained after recording anodic $i, \tau$-curves at $E=0.5$ and 0.9 in a solution with BTA were characterized by potential delays $E_{\mathrm{d}}$, Table 4 . The $E$, $\tau$-curves obtained in pure borate solution and in the presence of BTA are somewhat different. In a solution with the additive, two areas of potential delays remain only in the case of bismuth. The potential delays on the $E, \tau$-curves recorded for all the systems in the solution containing BTA are more negative than in pure borate. On the eutectic alloy, $E_{\mathrm{d}}$ has an intermediate value between the two $E_{\mathrm{d}}$ values found in pure borate solution.

Table 4. Parameters of chronoampero- and chronopotentiograms $\left(i_{\mathrm{c}}=-0.4 \mathrm{~A} / \mathrm{m}^{2}\right)$.

\begin{tabular}{|c|c|c|c|}
\hline \multirow{2}{*}{ Parameter } & \multicolumn{3}{|c|}{ Value of the parameter for $[\mathrm{Bi}]_{0}$, wt.\% } \\
\hline & 0 & 60 & 100 \\
\hline \multicolumn{4}{|c|}{$E=0.5 \mathrm{~V}$} \\
\hline$-E_{\mathrm{d} .0}, \mathrm{mV}$ & 717 & $683 ; 753$ & $544 ; 657$ \\
\hline$-E_{\text {d.add }}, \mathrm{mV}$ & 733 & 714 & $687 ; 743$ \\
\hline$K_{0}, \%$ & 7 & 37 & 29 \\
\hline$K_{\text {add }}, \%$ & 7 & 12 & 42 \\
\hline$\gamma_{q . i}$ & 1.5 & 0.6 & 1.3 \\
\hline$\gamma_{q . E}$ & 1.5 & 2.0 & 0.9 \\
\hline \multicolumn{4}{|c|}{$E=0.9 \mathrm{~V}$} \\
\hline$-E_{\mathrm{d} .0}, \mathrm{mV}$ & 718 & $698 ; 742$ & $624 ; 723$ \\
\hline$-E_{\text {d.add }}, \mathrm{mV}$ & 734 & 717 & $682 ; 733$ \\
\hline$K_{0}, \%$ & 6 & 42 & 69 \\
\hline$K_{\text {add }}, \%$ & 9 & 14 & 100 \\
\hline$\gamma_{q . i}$ & 2.0 & 0.9 & 1.2 \\
\hline$\gamma_{q . E}$ & 1.5 & 2.8 & 0.8 \\
\hline
\end{tabular}

Note: $K_{0}$ and $K_{\text {add }}$ are the ratios of the quantity of electricity consumed for the reduction and formation of the films. Indices ' 0 ' and 'add' refer to a solution without and with the additive, respectively. 
In a solution containing benzotriazole, the specific charge consumed for the formation of the passive film $q_{\mathrm{i}}$ on cadmium and on the alloy is much higher than that consumed to reduce it, $q_{\mathrm{E}}$. As could be expected, there is a greater difference in the ratio of $q$ values obtained in pure borate buffer and in the presence of BTA.

The share of current consumed for oxide film formation increases with an increase in $E$. In a solution with BTA it decreases on the alloy at $E=0.5 \mathrm{~V}$ and increases on bismuth. At $E=0.9 \mathrm{~V}$, the share of current consumed for the formation of oxide increases.

\section{Conclusion}

A similarity in the anodic behavior of the alloy and its electronegative component, i.e., cadmium, was observed in the solutions studied. The overall shape of the curve obtained on the alloy is similar to that constructed using the additive scheme; however, the currents on the experimental curve are lower in the presence of the additive.

Benzotriazole inhibits the dissolution of the metals and alloy upon direct and reverse polarization. In the case of cadmium and the alloy, adsorption of the additive on the surface causes a significant distortion of the polarization curve.

In the presence of the additive, the process occurs under diffusion and diffusionkinetic control. The share of current consumed to form the passive film is much higher than that of the current consumed to reduce it.

\section{References}

1. A. G. Berezhnaya and V. V. Ekilik, Korroz.: Mater., Zashch., 2007, no. 2, 6 (in Russian).

2. V. V. Ekilik, A. G. Berezhnaya and U. V. Dovbnya, Korroz.: Mater., Zashch., 2010, no. 6, 13 (in Russian).

3. N. D. Hobbins and R. F. Roberts, Surf. Technol., 1979, 9, 235.

4. R. Youda, H. Nishihara and K. Aramaki, Electrochim. Acta, 1990, no. 6, 1011.

5. A. G. Berezhnaya, V. I. Mishurov and V. V. Ekilik, Korroz.: Mater., Zashch., 2012, no. 4, 16 (in Russian).

6. S. N. Grushevskaya, T. A. Kuznetsova and A. V. Vvedensky, Zashch. Met., 2001, no. 6, 613 (in Russian). 